\author{
Kalyan Athmuri and Val R. Marinov \\ North Dakota State University, Industrial and Manufacturing Engineering, \\ Fargo,ND 58102,USA, val.marinov@ndsu.edu
}

\title{
OPTICALLY TRANSPARENT AND STRUCTURALLY SOUND SILICA AEROGELS: INSIGHTS FROM A PROCESS STUDY
}

\begin{abstract}
Aerogels are internally nanostructured materials characterized with a plethora of unique properties. Monoliths with high optical transparency made of silica aerogels were some of the first and still one of the most important classes of aerogels. Experiments and theory indicate that optical transparency and structural integrity of silica aerogels are negatively correlated. Other than optimal combination of processing conditions during aerogel fabrication can result in either highly transparent but cracked or in crack-free but less transparent and even opaque aerogels monoliths. Results are presented from the study of the relationship between the properties of single-step tetramethoxysilane (TMOS) base-catalyzed silica aerogels and the processing conditions, both at the alcogel preparation step and during the supercritical liquid $\mathrm{CO}_{2}$ drying process. Crack-free aerogel monoliths with good optical transparency were obtained with TMOS:methanol (MeOH) molar ratios of 1:16 and TMOS:ammonia $\left(\mathrm{NH}_{4} \mathrm{OH}\right)$ molar ratios of 1:0.05, $\mathrm{CO}_{2}-\mathrm{MeOH}$ exchange rates of about $1.25 \mathrm{ml} / \mathrm{min}$, and autoclave decompression rates of $70 \mathrm{KPa} / \mathrm{min}$. Adding glycerol in the sol-gel stage had a positive effect on the aerogel monolithicity but, even without glycerol, crack-free silica aerogels can be obtained by reducing the depressurization rate of the autoclave. A strict control and careful selection of the aerogel's processing conditions within the set of parameters identified will enable the fabrication of structurally sound silica aerogels with good optical properties essential for a number of applications.
\end{abstract}

Key words: silica aerogel; transparent aerogel monolith

\section{INTRODUCTION}

Aerogels are synthetic materials best defined as gels in which the liquid has been replaced by air with a very moderate shrinkage of the solid network [1]. Aerogels are characterized with unique morphology and material properties, including very high porosity (up to 99\%) and a sponge-like, three-dimensional open-pore structure with a wide-pore size distribution at the micro-, meso-, and macro scale. Their high porosity results in an extremely large inner surface area, which makes this material suitable for applications that can benefit from this property [2-5]. Some aerogels are known to possess one of the lowest thermal conductivities among all solids, rendering them suitable for a variety of insulation applications [6]. The experimental particle physics benefits from another property of the silica aerogels - their extremely low index of refraction close to that of air [7]. This property follows from their low apparent density through the Gladstone-Dale law, which for an optically isotropic material can be written as $n=1+k \rho$, where $n$ is the optical refractive index, $k$ is the refractive energy 
constant, $k_{\mathrm{SiO}_{2}}=0.208$ [8], and $\rho$ is the apparent density. These and some other unique properties and applications of aerogels are widely publicized [1, 9-11].

\section{Optical transparency of silica aerogels}

There are many types of aerogels, the most common and best studied of which are the silica aerogels, first synthesized in 1931 by Kistler [12]. The structure of the silica aerogel can be best described as a network of entangled and interconnected strings composed of agglomerates of silica particles. One of the unique properties of the silica aerogels is the possibility, first reported in 1968 by the Teichner group [13], to synthesize optically transparent monoliths using tetraalkoxysilanes $\mathrm{Si}(\mathrm{OR})_{4}$ as the silica source. The light absorption of silica in the visible range is negligible, therefore, the extinction of light should result from scattering due to imperfections on the aerogel surface and from bulk scattering from the aerogel structure [9]. The latter depends of the physical characteristics of the nanoscopic aerogel network of silica particles. Each aerogel has its own structural characteristics but on the average, the primary colloidal particles that form the pore structure of the silica aerogels have a diameter of only $1-3 \mathrm{~nm}$ and the mean pore diameter is $20-150$ nm [11]. Therefore, most of the optical properties of the silica aerogels can be explained with the Rayleigh-Gans approximate solution to light scattering, which is pertinent to the case when the relative refractive index of the particle (in case of silica aerogels, approximated with a $\mathrm{SiO}_{2}$ sphere) is close to unity, and its size is less than $10 \%$ of the wavelength of the visible light. Because visible light has wavelength between 400 and $800 \mathrm{~nm}$, the Rayleigh-Gans approximation would be applicable to particle sizes or particle agglomerates of less than 40 $\mathrm{nm}$, which condition is, in general, satisfied for most silica aerogels. Although the RayleighGans approximation was developed for a single isolated particle in an ideal medium, it was shown that it can be used in the analysis of aerosol scattering data, particularly in the nearforward direction [14]. There is evidence that this is true for silica aerogels as well $[9,15]$. The physical basis of the Rayleigh-Gans approximation is discussed in detail in [16] and is beyond the scope of this discussion. What is important for us is that the Rayleigh-Gans approximation, when applied to silica aerogels, implies that the extinction due to bulk scattering, ergo, the attenuation of the transmitted visible light, increases with the $\mathrm{SiO}_{2}$ particles size. Consequently, to achieve high optical transparence, the particle (and thus the agglomerate) sizes have to be kept small. The aerogel structure, including the particle size, can be controlled by manipulating polymerization conditions in solution precursors [17], the molar ratio of the constituent materials used to prepare the alcogel, the type of precursors used, and the parameters of the aging process, in particular, the process of Oswald ripening or coarsening $[18,19]$.

\section{Structural integrity of transparent silica aerogels}

Experiments with transparent aerogels have indicated that in most cases transparency and structural integrity of the material are inversely correlated. Other than optimal sol-gel parameters during aerogel fabrication can lead to either transparent but cracked monoliths, or to crack-free but less transparent and even opaque aerogels [20, 21]. However, even if the sol-gel parameters were optimized the aerogels may still crack during the supercritical drying 
process. The pressure buildup inside the network of pores, resulting from allowing the pressure gradient across the aerogel to increase too much, is responsible for this phenomenon. The pressure gradient depends on the characteristics of the fluid flow through the aerogel pores [22]. It also depends on the aerogel structure. In spite of their network of open pores, aerogels are characterized with a very low permeability [23]. The aerogel permeability and the rate at which the supercritical fluid is released from the autoclave control the pressure gradient across the aerogel, and from there, the structural integrity of the monolith. Gas permeability of aerogels is governed by largest pores and evolves in the same way as the pore size distribution [24]. Large pores and an open pore structure are required to maintain high permeability. Conversely, and as discussed before, optical transparency is governed by Rayleigh scattering, which postulates that transmittance decreases with the size of particles and pores. Therefore, an attempt to improve structural integrity by increasing aerogel permeability would have a negative effect on the optical transparency, something that we have observed in our experiments as well. Strengthening the aerogel structure by promoting the Oswald ripening during the alcogel aging step will have a similar detrimental effect.

Another important factor is the diffusion kinetics of the liquid $\mathrm{CO}_{2}-$ solvent exchange in the autoclave during the initial stages of the supercritical $\mathrm{CO}_{2}$ drying process. It was shown [25, 26] that the structural damage at this point is due to unsteady-state diffusion of solvent and liquid $\mathrm{CO}_{2}$, which occurs when the solvent and liquid $\mathrm{CO}_{2}$ mixture is below the binary critical curve where both liquids are not miscible and exist as two separate phases. The results is that some of the solvent will remain inside the aerogel after the $\mathrm{CO}_{2}$ - solvent exchange and, if its concentration is high enough, the mixture would not reach supercritical state defined by the supercritical point of the neat $\mathrm{CO}_{2}$ liquid. Eventually, the supercritical drying will not be able to eliminate the capillary forces in the pores and the fragile aerogel structure will collapse.

Under the right conditions in the $\mathrm{CO}_{2}$ - solvent exchange, the liquid $\mathrm{CO}_{2}$ surrounding the alcogel in the autoclave and the solvent that fills its pores are miscible. It's well known that when two miscible liquids are brought into contact, diffusion takes place. Fick's first law of diffusion can be rewritten as $J \propto-P\left(c_{2}-c_{1}\right)$, where $J$ is the rate at which solvent diffuses out through a unit area of the alcogel, $P$ is the alcogel permeability, and $c_{2}-c_{1}$ is the difference in concentration of the solvent across the aerogel in the direction of flow. Since the permeability of alcogels is very low, it follows that the diffusion flux will be very low, too, especially, by the end of the exchange time when the concentration gradient is small. Therefore, sufficient time should be provided for the organic solvent held within the alcogel's pores to diffuse out and the liquid $\mathrm{CO}_{2}$ to diffuse in its place. Otherwise the solvent will remain entrapped in the pores of the alcogel with all the negative effects discussed in the previous paragraph.

In summary, it appears that some of the most critical factors that control the structural integrity of silica aerogels are related to the rates in the supercritical $\mathrm{CO}_{2}$ drying process, including the rate of $\mathrm{CO}_{2}$ - solvent exchange and the vessel depressurization rate during drying. Pajonk and co-workers [19] have studied the effect of some other supercritical drying conditions such as the volume of the solvent filled in the autoclave, the initial pre-pressure in the autoclave, the heating rates, and the stabilization period.

In spite of its importance, the relationship between the optical transparency and the structural integrity of silica aerogel monoliths in the context of the processing conditions has not been studied extensively. The present work is an attempt to fill this gap and to provide insights into this relationship in case of tetramethoxysilane (TMOS) base-catalyzed silica aerogels. 


\section{MATERIALS AND METHODS}

\section{Sample preparation}

Since 1962, silica aerogels are commonly synthesized by a single-step, base-catalyzed polymerization of alkoxides such as tetraethoxysilane (TEOS) or TMOS used to prepare via the sol stage the starting alcogel. The pores of the alcogel at this stage are filled mainly with water and/or alcohol. The alcogel is then dried to replace this liquid phase with air, leaving behind the characteristic sponge-like solid structure of the aerogel. The drying step is typically accomplished by the so-called low-temperature supercritical drying in liquid $\mathrm{CO}_{2}$. This process was developed in the early 1980s by Hunt, Tewari and co-workers from the Lawrence Berkeley Laboratory [27] as a safer and more efficient alternative to the originally used drying in organic solvents. This and other routes for fabrication of silica aerogels are well publicized; therefore, a detailed discussion on this topic is not presented here.

All alcogels in this study were prepared by hydrolysis and condensation of methanol-diluted TMOS in the presence of a basic catalyst (ammonia, $\mathrm{NH}_{4} \mathrm{OH}$ ). The choice of TMOS was dictated by the fact that, as far as the monolithicity and optical transparency are concerned, the TMOS aerogels are superior compared to the TEOS aerogels [28]. As mentioned before, the transparency of the silica aerogels mostly depends on the molar ratio of the starting materials. In the first series of experiments, alcogels with different molar ratios were prepared and dried without varying the parameters of the supercritical $\mathrm{CO}_{2}$ drying process. The objective was to identify these alcogel preparation conditions that would result in silica aerogels with high optical transparency and minimal if any amount of cracking. No attempt was made in these experiments to optimize the drying conditions in order to completely eliminate the cracking in the aerogel. This was the objective for the next series of experiments.

In addition to the differences in the starting material compositions, samples were treated differently during the $\mathrm{CO}_{2}$ exchange. For the samples with compositions listed in Table 1, the $\mathrm{CO}_{2}$ exchange was carried out gradually as explained in the next paragraph. The $\mathrm{CO}_{2}$ exchange rate was much faster for the samples in Table 2.

Table 1. Molar ratios used in the slow $\mathrm{CO}_{2}$ exchange experiments

\begin{tabular}{cccc}
\hline TMOS & $\mathrm{H}_{2} \mathrm{O}$ & $\mathrm{MeOH}$ & $\mathrm{NH}_{4} \mathrm{OH}$ \\
\hline \hline 1 & 4 & 8 & 0.005 \\
1 & 4 & 12 & 0.005 \\
1 & 4 & 16 & 0.005 \\
1 & 4 & 8 & 0.025 \\
1 & 4 & 12 & 0.025 \\
1 & 4 & 16 & 0.025 \\
1 & 4 & 8 & 0.05 \\
1 & 4 & 12 & 0.05 \\
1 & 4 & 16 & 0.05 \\
\hline
\end{tabular}

Table 2. Molar ratios used in the quick $\mathrm{CO}_{2}$ exchange experiments

\begin{tabular}{cccc} 
TMOS & $\mathrm{H}_{2} \mathrm{O}$ & $\mathrm{MeOH}$ & $\mathrm{NH}_{4} \mathrm{OH}$ \\
\hline \hline 1 & 4 & 8 & 0.005 \\
1 & 4 & 16 & 0.005 \\
1 & 4 & 8 & 0.05 \\
1 & 4 & 16 & 0.05 \\
\hline
\end{tabular}


The detailed procedure for aerogels preparation is explained elsewhere [29]; only a brief description is provided here. The silica alcogels were prepared by mixing TMOS (99\% purity, Sigma Aldrich), deionized water, $\mathrm{MeOH}$ (HPLC, Mallinckrodt Reagent Chemicals) and $\mathrm{NH}_{4} \mathrm{OH}(28-30 \%, V W R)$ in 1 in. (2.54 mm) diameter cylindrical polypropylene molds. The total volume of the solution in each mold was $5 \mathrm{ml}$. Once the alcogels were set, methanol was poured on top of the alcogel for aging and the molds were closed airtight to avoid the evaporation of $\mathrm{MeOH}$. The alcogels were allowed to age for $72 \mathrm{~h}$.

After aging, the alcogels were removed from their molds and soaked in ethanol for curing. The ethanol bath was replaced twice a day for 3 days. After the curing was completed, the alcogels were loaded in the autoclave. The sizes of the alcogels at that point were about 20-22 $\mathrm{mm}$ in diameter and $11-13 \mathrm{~mm}$ in thickness. The autoclave was immediately filled with ethanol until the ethanol level rose about $20 \mathrm{~mm}$ above the gels. The ethanol was replaced with liquid $\mathrm{CO}_{2}$ (bone dry, Airgas). For the quick rate of $\mathrm{CO}_{2}$ exchange experiments (specimens in Table 2) the buffer ethanol in the autoclave was removed in a single step within $1.5 \mathrm{~h}$ after loading the gels at a rate of $6 \mathrm{ml} / \mathrm{min}$, whereas for the low rate of $\mathrm{CO}_{2}$ exchange experiments (specimens in Table 1) the ethanol was gradually exchanged with liquid $\mathrm{CO}_{2}$ over a period of $8 \mathrm{~h}$, which resulted in an exchange rate of about $1.25 \mathrm{ml} / \mathrm{min}$. After the removal of ethanol, the liquid $\mathrm{CO}_{2}$ in the autoclave was replaced twice a day until no more ethanol was recovered, and then two extra liquid $\mathrm{CO}_{2}$ exchanges were carried out to ensure that not even a small amount of ethanol remained in the autoclave. Finally, the aerogel samples were obtained from the alcogels following the conventional procedure for supercritical $\mathrm{CO}_{2}$ drying. The chamber depressurization rate in these experiments was 140 $\mathrm{kPa} / \mathrm{min}$.

Table 3. Molar ratios used in the slow depressurization rate experiments

\begin{tabular}{ccccc}
\hline TMOS & $\mathrm{H}_{2} \mathrm{O}$ & $\mathrm{MeOH}$ & $\mathrm{NH}_{4} \mathrm{OH}$ & Glycerol \\
\hline \hline 1 & 4 & 12 & 0.05 & 0 \\
1 & 4 & 12 & 0.05 & 0.05 \\
1 & 4 & 12 & 0.05 & 0.2 \\
1 & 4 & 12 & 0.05 & 0.4 \\
1 & 4 & 12 & 0.05 & 0.6 \\
1 & 4 & 12 & 0.05 & 0.8 \\
\hline
\end{tabular}

In order to study the effects of the rate of depressurization in the supercritical $\mathrm{CO}_{2}$ drying process on the structural integrity of TMOS silica aerogels, another series of aerogel samples was prepared using the optimal sol-gel parameters identified in the previous experiments. Additionally, glycerol was added to some of the samples in this group to act as a drying control chemical additive (DCCA), which has been reported to be effective in preventing cracking in silica aerogels [30]. Eighteen aerogel samples were prepared with TMOS: $\mathrm{H}_{2} \mathrm{O}: \mathrm{MeOH}: \mathrm{NH}_{4} \mathrm{OH}$ molar ratio of 1:4:12:0.05 and 6 different concentrations of glycerol, as shown in Table 3. Each formulation was repeated 3 times for a total of 18 samples. All alcogels were dried together using the same parameters as these for the samples in Table 1 but with a slower depressurization rate of $70 \mathrm{KPa} / \mathrm{min}$.

The fabrication of silica aerogels requires a meticulous and skillful preparation and a strict control of a number of variables. Even though, a lot of things may go wrong and the end 
results might be quite different from what was expected. The challenges prompted Sorensen [31] to comment in her thesis, “...the creation of a gel is not always reproducible. What seem to be identical gels created at different times can greatly vary ... once allowed to dry.” The authors must agree with this statement since similar observations were made in our experiments. This may be the reason for some of the randomness in the results discussed in the next section.

\section{Characterization}

The optical transparency of the aerogels was measured using a UV-2501PC UV-VIS spectrophotometer (Shimadzu) at various points at an increment of $0.5 \mathrm{~nm}$ over the range from $200 \mathrm{~nm}$ to $800 \mathrm{~nm}$. The values for the optical transmittance were used to construct transmittance curves. The average transmittance for each curve was calculated by numerically integrating the area under the transmittance curve over the visible range of light $(400 \mathrm{~nm}$ $700 \mathrm{~nm})$.

The structural integrity of the samples was evaluated by measuring the total area of the cracked surfaces. No attempt was made to distinguish between the size and the number of cracks, i.e., a single crack with a large fracture area yielded the same result as many smaller cracks with a cumulative fracture surface area equal to this of the larger crack. Pictures were taken for each aerogel sample from three sides, as shown in Fig. 1.

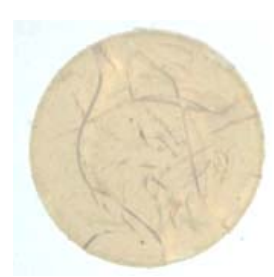

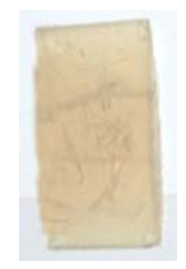

(a)

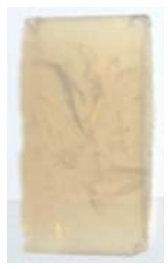

Fig. 1. (a) Top view, side view, and side view with 90 deg. rotation of a cracked aerogel sample; (b) a black-andwhite top view of the same aerogel after image processing

Image processing software was used to convert the color digital photographs to black-andwhite images in which the cracks appeared as black areas (Fig. 1b). Another software package (Pixcavator $^{\circledR}$ ) was used to analyze the black-and-white images and extract information about the total area of the cracks in all three views. The measurements were then averaged and presented as the mean crack area of the sample.

\section{RESULTS AND DISCUSSION}

\section{Aerogel transparency}

The transparency values for the different aerogels for each combination of TMOS: $\mathrm{NH}_{4} \mathrm{OH}$ and TMOS:MeOH molar ratios obtained by a slow $\mathrm{CO}_{2}$ exchange rate are shown in Fig. 2. 
The results from the specimens in Table 2 in which the $\mathrm{CO}_{2}$ exchange rate was much higher are compared with the results obtained with a low $\mathrm{CO}_{2}$ exchange rate in Fig. 3.

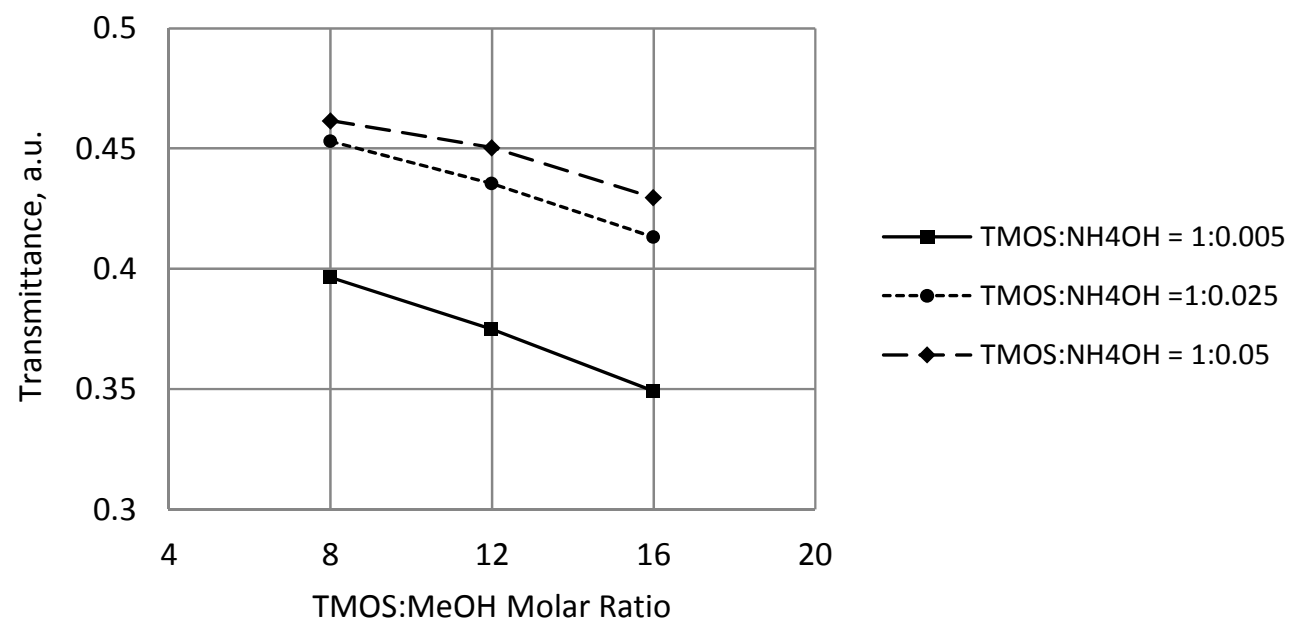

Fig. 2. Effect of the starting material composition on the silica aerogel transmittance for specimens prepared with slow $\mathrm{CO}_{2}$ exchange rates

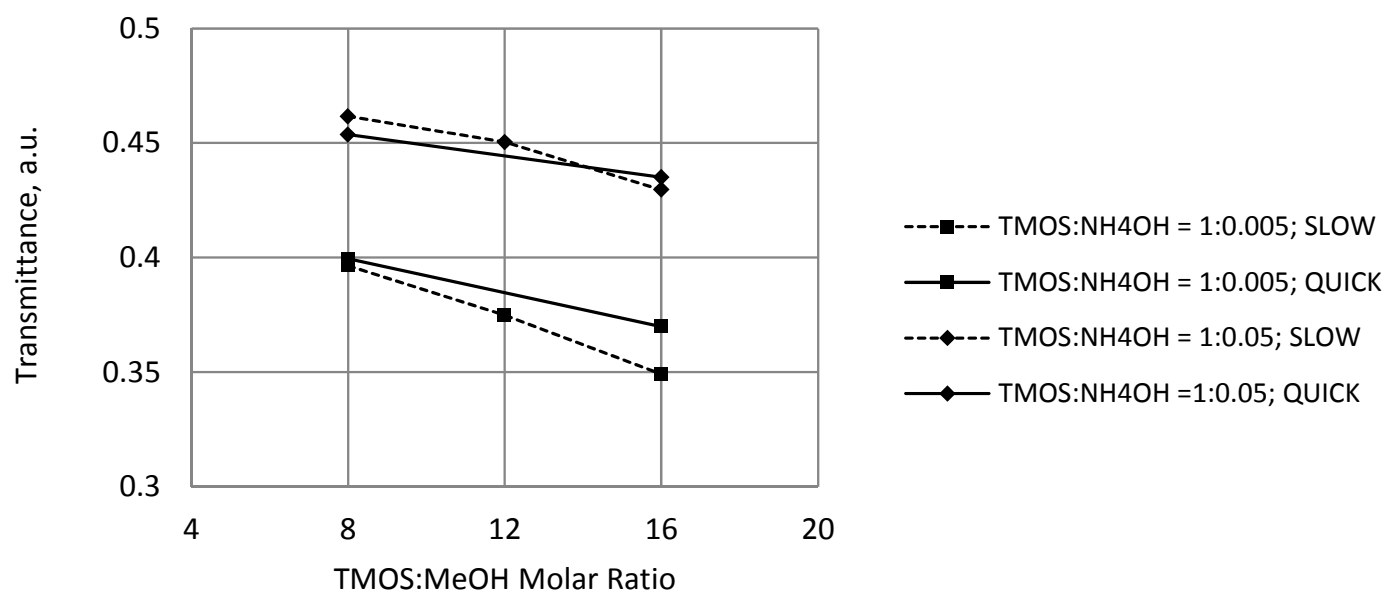

Fig. 3. Transmittance of silica aerogels prepared with slow and quick $\mathrm{CO}_{2}$ exchange rates

Fig. 2 demonstrates a clear relationship regarding the effects of the TMOS:MeOH and TMOS: $\mathrm{NH}_{4} \mathrm{OH}$ molar ratios on the transparency of the aerogels. Similar results were reported by Rao et al. [21]. The effect of ammonia is much more pronounced at higher TMOS: $\mathrm{NH}_{4} \mathrm{OH}$ ratios. The lower transparency at low TMOS: $\mathrm{NH}_{4} \mathrm{OH}$ ratios can be attributed to the fact that no sufficient amount of catalyst was available in the alcogel pores to complete the hydrolysis and condensation reactions, leading to a formation of large clusters of particles [21], and therefore, to a larger light scattering. On the other hand, Fig. 3 shows that no significant difference was observed between the transparency values for the aerogels obtained by slow and quick $\mathrm{CO}_{2}$ exchange rates, especially, at higher TMOS: $\mathrm{NH}_{4} \mathrm{OH}$ ratios. Surprisingly, the quick $\mathrm{CO}_{2}$ exchange rate resulted in aerogels with higher optical transmittance when the TMOS: $\mathrm{NH}_{4} \mathrm{OH}$ ratio was small. Obviously, the optical properties of the aerogels are more 
sensitive to the processing conditions when the amount of catalyst is small compared to the amount of reactants.

The transmittance values for aerogels prepared with addition of various concentrations of glycerol and a constant molar ratio of TMOS: $\mathrm{H}_{2} \mathrm{O}: \mathrm{MeOH}: \mathrm{NH}_{4} \mathrm{OH}$ molar ratio of 1:4:12:0.05 are shown in Fig. 4.

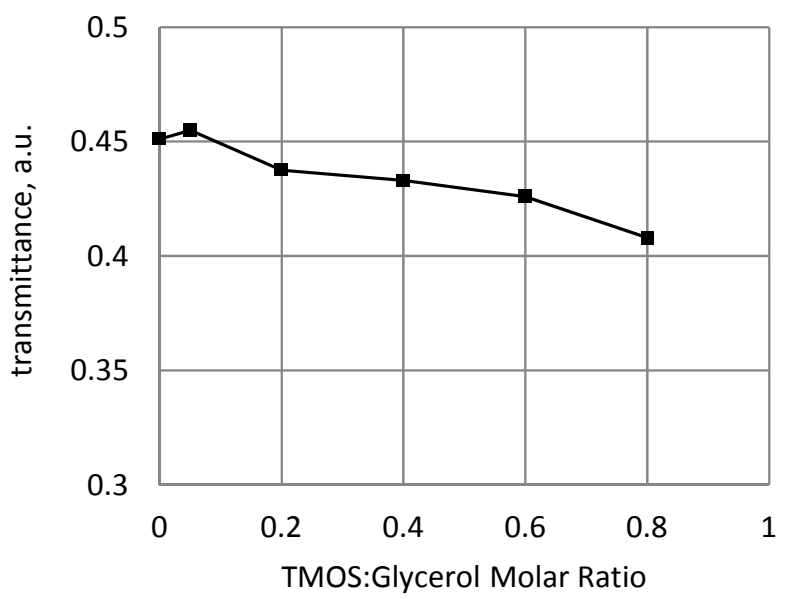

Fig. 4. Transmittance of silica aerogels prepared with different concentrations of glycerol (Table 3)

The results in Fig. 4 indicate that adding glycerol up to about 0.1 TMOS:glycerol ratio affects negligibly the aerogel transparency. Increasing this ratio has a certain detrimental effect on the optical transparency, which seems to intensify with the amount of glycerol added. Similar results were reported by Rao and Kulkarni [32] who hypothesized that the decrease in the optical transmission at higher TMOS:glycerol molar ratios is due to the formation of irregular pores caused by random distribution of glycerol in the loose network of siloxane chains.

\section{Aerogel monolithicity}

The crack measurements for each aerogels obtained by slow $\mathrm{CO}_{2}$ exchange rate are shown in Fig. 5. No measurements were taken from the aerogels obtained by quick $\mathrm{CO}_{2}$ exchange rate. All of the specimens in this case appeared extremely cracked, which once again emphasizes the importance of controlling the rate of $\mathrm{CO}_{2}$ - solvent exchange. Increased cracking in silica aerogels as a result of the quick diffusion of ethanol from gels into the liquid $\mathrm{CO}_{2}$ was also observed in other studies [25, 33]. 


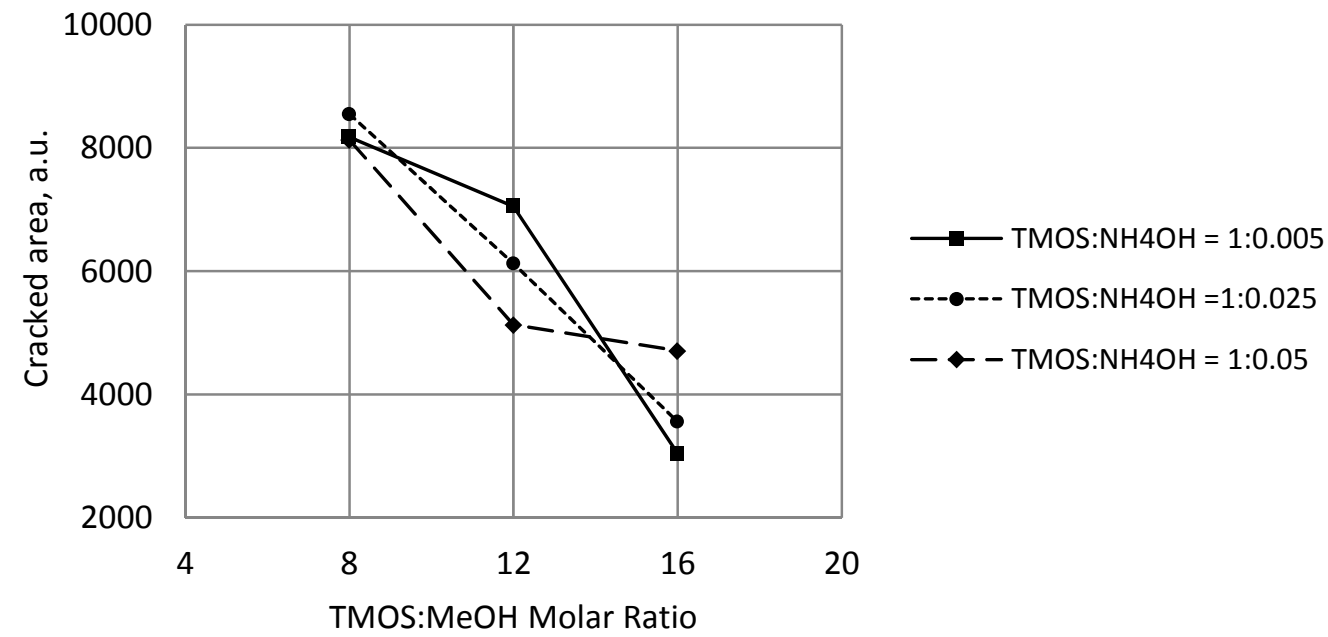

Fig. 5. Effect of the starting material composition on the silica aerogel monolithicity for specimens prepared with a slow $\mathrm{CO}_{2}$ exchange rate. The cracked area of each sample was calculated by averaging the results from three photographs as shown in Fig. 1a. The measurement was done by counting the black pixels in the black-and-white images similar to this shown in Fig. $1 \mathrm{~b}$

Although the effect of the TMOS:MeOH molar ratio on the aerogel monolithicity is quite obvious, no such relationship was observed if the TMOS: $\mathrm{NH}_{4} \mathrm{OH}$ ratios are considered. One possible explanation might be the approximations introduced by the method used in this study to measure the integrity of the aerogel. Nonetheless, this method gives us a chance to quantify, at least approximately, the amount of cracking within the aerogel structure, something which, to the best of authors' knowledge, has not been reported before.

The tradeoff between optical transmittance and structural integrity is clearly visible when comparing the results in Figs. 2 and 5. The best optical transmittance in the visible spectrum is achieved at lower TMOS:MeOH ratios and higher TMOS: $\mathrm{NH}_{4} \mathrm{OH}$ ratios. This, according to the results in Fig. 5, is precisely the combination that should result in the most cracked aerogels.

During the second series of experiments, decreasing the decompression rate from 140 $\mathrm{KPa} / \mathrm{min}$ to $70 \mathrm{KPa} / \mathrm{min}$ resulted in crack-free aerogels in $61 \%$ of the aerogel samples. The cracks in the rest of the samples were hardly visible and much less than the cracks in the aerogels depressurized at the higher rate. Fig. 6 shows an example of a no-glycerol, crackfree, transparent aerogel monolith obtained with a depressurization rate of $70 \mathrm{KPa} / \mathrm{min}$.

Perhaps the most surprising result from the second series of experiments in which glycerol was added to the sol during the sol-gel preparation stage was that there was no detectable difference in monolithicity between the aerogels with and without glycerol. Adding a DCCA like glycerol might be helpful in producing crack-free aerogels as reported in the literature [32], but our results indicated that the same outcome can be achieved by simply decreasing the rate of pressure release from the autoclave. 


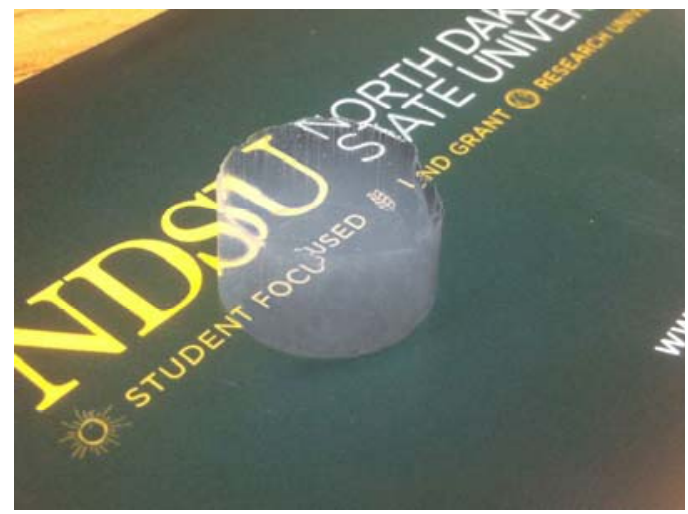

Fig. 6. Photograph showing an example of a transparent and crack-free silica aerogel in this study. The small piece of the aerogel missing was accidentally separated from the alcogel when it was removed from the mold

\section{CONCLUSIONS}

Monolithic silica aerogels with high optical transparency were synthesized following the one-step TMOS base-catalyzed routine followed by supercritical $\mathrm{CO}_{2}$ drying. Aerogels with good optical transparency were obtained with TMOS:MeOH molar ratios of 1:16 and TMOS: $\mathrm{NH}_{4} \mathrm{OH}$ molar ratios of 1:0.05. The optical properties of the silica aerogels were unaffected by the rate of solvent- $\mathrm{CO}_{2}$ exchange and the autoclave decompression rate. However, it was found that these two parameters affected significantly the monolithicity of the silica aerogels. Monolithic aerogels with no cracks were obtained with $\mathrm{CO}_{2}$ exchange rate of about $1.25 \mathrm{ml} / \mathrm{min}$ and a decompression rate of $70 \mathrm{KPa} / \mathrm{min}$. Adding glycerol to the starting solution had a positive effect on the aerogel monolithicity but no-glycerol, crack-free silica aerogels were also obtained by reducing the depressurization rate of the autoclave from $140 \mathrm{KPa} / \mathrm{min}$ to $70 \mathrm{KPa} / \mathrm{min}$. The silica aerogel monolithicity was also affected by the solgel processing conditions. It was found that there is a negative relationship between the parameters that lead to highly optically transparent silica aerogels and these that resulted in crack-free monoliths. For example, increasing the TMOS:MeOH molar ratios from 1:8 to 1:16 resulted in more transparent but also more cracked aerogels.

\section{REFERENCES}

1. Aegerter M.A., Leventis N., Koebel M.M.: Aerogels Handbook. Springer, New York, 2011.

2. Hirashima H., Kojima C., Imai H.: Application of alumina aerogels as catalysts. Journal of SolGel Science and Technology 8 (1997), 843-846.

3. Smirnova A., Dong X., Hara H., Vasiliev A., Sammes N.: Novel carbon aerogel-supported catalysts for PEM fuel cell application. International journal of hydrogen energy 30 (2005), 149158.

4. Moreno-Castilla C., Maldonado-Hódar F.: Carbon aerogels for catalysis applications: An overview. Carbon 43 (2005), 455-465. 
5. Pajonk G.: Aerogel catalysts. Applied Catalysis 72 (1991), 217-266.

6. Smith D.M., Maskara A., Boes U.: Aerogel-based thermal insulation. Journal of non-crystalline solids 225 (1998), 254-259.

7. Cantin M., Casse M., Koch L., Jouan R., Mestreau P., Roussel D., Bonnin F., Moutel J., Teichner S.: Silica aerogels used as Cherenkov radiators. Nuclear Instruments and Methods 118 (1974), 177-182.

8. Mandarino J.: The Gladstone-Dale relationship. Part I: Derivation of new constants. Canadian Mineralogist 14 (1976), 498-502.

9. Emmerling A., Petricevic R., Beck A., Wang P., Scheller H., Fricke J.: Relationship between optical transparency and nanostructural features of silica aerogels. Journal of non-crystalline solids 185 (1995), 240-248.

10. Fricke J.: Aerogels. Springer-Verlag, Berlin, 1986.

11. Hüsing N., Schubert U.: Aerogels-Airy Materials: Chemistry, Structure, and Properties. Angewandte Chemie International Edition 37 (1998), 22-45.

12. Kistler S.: Coherent Expanded Aerogels and Jellies. Nature 127 (1931), 741.

13. Nicolaon G., Teichner S.: New preparation process for silica xerogels and aerogels, and their textural properties. Bulletin de la Société Chimique de France 5 (1968), 1900-1906.

14. Box M., Lo S., McKellar B., Reich M.: The application of the Rayleigh-Gans approximation to scattering by polydispersions. Quarterly Journal of the Royal Meteorological Society 104 (1978), 959-969.

15. Beck A., Gelsen O., Wang P., Fricke J.: Light scattering for structural investigations of silica aerogels and alcogels. Le Journal de Physique Colloques 50 (1989), 4-4.

16. Van de Hulst H.: Light scattering by small particles. Dover Publications, Mineola, N.Y., 1981.

17. Schaefer D.: What factors control the structure of silica aerogels? Le Journal de Physique Colloques 50 (1989), 4-4.

18. Voorhees P.: The theory of Ostwald ripening. Journal of Statistical Physics 38 (1985), 231-252.

19. Pajonk G., Venkateswara Rao A., Sawant B., Parvathy N.: Dependence of monolithicity and physical properties of TMOS silica aerogels on gel aging and drying conditions. Journal of noncrystalline solids 209 (1997), 40-50.

20. Pajonk G.M.: Some applications of silica aerogels. Colloid \& Polymer Science 281 (2003), 637651.

21. Venkateswara Rao A., Pajonk G., Haranath D., Wagh P.: Effect of sol-gel processing parameters on optical properties of TMOS silica aerogels. Journal of Materials Synthesis and Processing 6 (1998), 37-48.

22. Scherer G.W.: Theory of drying. Journal of the American Ceramic Society 73 (1990), 3-14.

23. Hosticka B., Norris P., Brenizer J., Daitch C.: Gas flow through aerogels. Journal of noncrystalline solids 225 (1998), 293-297.

24. Calas S., Sempere R.: Textural properties of densified aerogels. Journal of non-crystalline solids 225 (1998), 215-219.

25. Rogacki G., Wawrzyniak P.: Diffusion of ethanol-liquid CO2 in silica aerogel. Journal of noncrystalline solids 186 (1995), 73-77.

26. Masmoudi Y., Rigacci A., Ilbizian P., Cauneau F., Achard P.: Diffusion during the supercritical drying of silica gels. Drying technology 24 (2006), 1121-1125. 
27. Tewari P., Hunt A., Lofftus K.: Ambient-temperature supercritical drying of transparent silica aerogels. Materials Letters 3 (1985), 363-367.

28. Wagh P., Begag R., Pajonk G., Rao A.V., Haranath D.: Comparison of some physical properties of silica aerogel monoliths synthesized by different precursors. Materials chemistry and physics 57 (1999), 214-218.

29. Athmuri K.: Transparent and Crack-free Silica Aerogels. M.Sc. Thesis, North Dakota State University, 2012.

30. Haranath D., Rao A.V., Wagh P.: Influence of DCCAs on optical transmittance and porosity properties of TMOS silica aerogels. Journal of Porous Materials 6 (1999), 55-62.

31. Sorensen L.M.: Embedding Luminescent Nanocrystals in Silica Sol-Gel Matrices. M.Sc. Thesis, Florida State University, 2006.

32. Rao A.V., Kulkarni M.M.: Effect of glycerol additive on physical properties of hydrophobic silica aerogels. Materials chemistry and physics 77 (2003), 819-825.

33. Novak Z., Knez Ž.: Diffusion of methanol-liquid CO2 and methanol-supercritical CO 2 in silica aerogels. Journal of non-crystalline solids 221 (1997), 163-169. 